\title{
EXECUTIVE SUMMARY \\ Public Discourses and Engagement on Governance of Covid-19 Response in Ekiti State, Nigeria
}
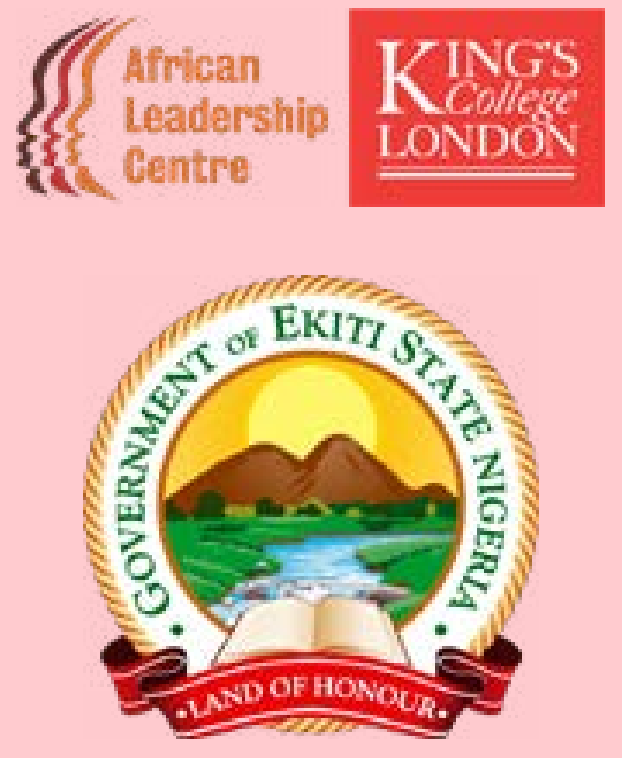

\section{December 2021}

(i) RESEARCHERS

Damilola Adegoke, PhD. (Project Leader/Lead Author), Research Associate and Head of the African Leadership Centre (ALC) Datalab, King's College London

Natasha Chilambo, ALC Research Associate

Adeoti Dipeolu, Research Associate and Doctoral Candidate, ALC, King's College London

Ibrahim Machina Mohammed, Peace, Security and Development Fellow, ALC, King's College London

Ade Obafemi-Olopade, Research Associate and Doctoral Candidate, ALC, King's College London

Dolapo Yusuf, Project Associate, ALC Datalab

RESEARCH ASSISTANT

Stephen Domingo, ALC Datalab

Download Full Report

DOI: 10.47697/lab.202101 


\section{Executive Summary}

\section{ABOUT THE RESEARCH}

Numerous studies have emerged so far on Covid-19 (SARS-CoV-2) across different disciplines. There is virtually no facet of human experience and relationships that have not been studied. In Nigeria, these studies include knowledge and attitude, risk perception, public perception have also been carried out on public framing of Covid-19 discourses in Nigeria; these have explored both offline and online messaging and issues from the perspectives of citizens towards government's policy responses such as palliative distributions, social distancing and lockdown. The investigators of these thematic concerns deployed different methodological tools in their studies. These tools include policy evaluations, content analysis, sentiment interviews as well as machine learning

These studies nearly always focus on the national government policy response, with little or no focus on the constituent states. In many of the studies, the researchers work with newspaper articles for analysis of public opinions while others use social media generated contents such as tweets) as sources for analysis of sentiments and opinions. Although there are others who rely on the use of survey questionnaires and other tools outlined above; the limitations of these approaches necessitated the research plan adopted by this study.

Most of the social media users in Nigeria are domiciled in cities and their demography to internet technologies. Hence, the opinions of a majority of the population who are most likely rural dwellers with limited access to internet technologies are very often excluded. This is not in any way to disparage social media content analysis findings; because the opinions expressed by opinion leaders usually represent the larger subset of opinions prevalent in the society. Analysing public perception using questionnaires is also fraught with its challenges, as well as reliance on newspaper articles. A lot of the newspapers and news media organisation in Nigeria are politically hinged, some of them have active politicians and their associates as their proprietors. Getting unbiased opinions from these sources might be difficult. The news interviews which usually privilege elite actors.

These gaps motivated this collaboration between Ekiti State Government and the African Leadership Centre at King's College London to embark on research that will primarily assess of the study covers the first phase of the pandemic in Ekiti State (March/April to August 2020). Todd, A., Akhter, N., Cairns, J. M., Kasim, A., Walton, N., Ellison, A., ... \& Bambra, C. (2018). The Pain Ufua, D. E., Osabuohien, E., ogbari, M. E., Falola, H. O., Okoh, E. E., \& Lakhani, A. (2021). Re-Strategsing Government Palliative Support Systems in Tackling the Challenges of Covid-19 Lockdown in Lagos State, Adamu, H., Lutfi, S. L., Malim, N. H. A. H., Hassan, R., Di Vaio, A., \& Mohamed, A. S. A. (2021). Framing
twitter Public Sentiment on Nigerian Government COVID-19 Palliatives Distribution Using Machine Learning. Sustainability, $13(6), 3497$.

\section{SUMMARY OF METHODOLOGIES}

\section{AND METHODS}

To achieve the key objectives and to address some of the gaps missed by existing literature on the subject of interest, we used a multi-pronged approach involving multiple methods. This research is splting four independent sub-studies. The first part in Ekiti State using Facebook Data for Good datasets and QGIS.

The second part explores social media analysis of the public perception of Government Covid-19 response in Ekiti State using quantitative content analysis. The third part assesses public perceptions and opinions on Covid-19 governance in Ekiti State through traditional news media using content analysis, analysis.

The final part examines public perspectives and opinion dimensions on government leadership response to Covid-19 More explanations for each of the methodologies and methods used in this research report are provided in the various study parts.

\section{SUMMARY OF KEY FINDINGS}

\section{Spatial and vulnerability mapping of at-risk people in \\ Ekiti State}

Part of the measures to control the spread of Covid-19 in Ekiti and Nigeria during the first phase of the pandemic outbreak was of the pattern of movement of the have a polations (Elderly: ages 60+) in the state, we used the Facebook Data for Good datasets which include Facebook's high-resolution population density data, Facebook's movement range dataset, and GADM shapefil polygon dataset together with QGIS 3.18.2 and the GRID3

\section{Key Research Objectives}

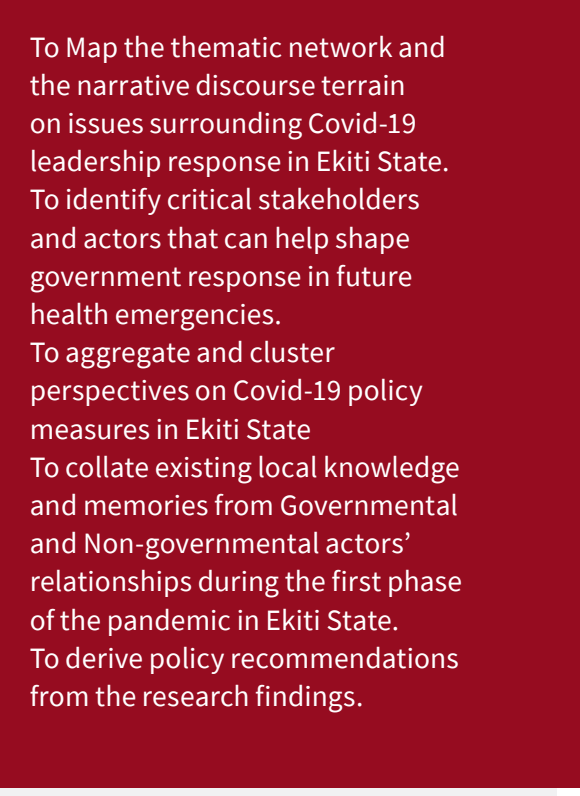

GIS database. ${ }^{6,7}$ These datasets and tools helped to do Covid-19 risk-vulnerability Ekiti state during the timeframe of study (i.e., between April and August of 2020).

Facebook's movement range data maps provide information about population response to physical social distancing measures using two metrics: Change in Movement and Stay Put. The former presents 'how much people are moving around and compares it with a baseline distancing predates most social 'looks at the fraction of the population that appear to stay within a small area during an entire day. ,, $9^{2}$ We also used the Geo-Referenced Infrastructure 6 Humanitarian Data Exchange. (2020). Facebook's Data for Good. Retrieved 31 August 2020, GRID3 (n.d.). Ekiti Health Care Facilities
Primary, Secondary and Tertiary). Retrieved 25 July (Primary, Secondary and Tertiary). Retrieved 25 July
2020 , from https://grid3.gov.ng/dataset/ekiti-health-care-facilities-primary-secondary-and-tertiary\# 8 Humanitarian Data Exchange. (2020). Mo-
8 vement Range Maps. Retrieved 31 August 2020, from
https://data.humdata.org/dataset/movement-ran9 ge-maps? 
and Demographic Data for Development (GRID3) for GIS shapefiles on Ekiti State health facilities and infrastructure. One
limitation of the Facebook Movement is that it depends on user activation of their location and other proximity features; hence, most rural areas are excluded from the data capture. To this end, Only Irepodun/ Ifelodun Local Government and Ado-Ekiti enough Facebook mobility data for the risk vulnerability analysis. Ado-Ekiti is the capital city of Ekiti State.

The collection of maps in figure 2 (on pp. 1920) depict the Covid-19 vulnerability score for at-risk individuals (age 60+) in Ado-Ekiti an Irepodun/Felodun local government areas of Ekiti state between March and August of 2020. Between March and April 2020, the risk and (mildly high: 60-69) for Irepodun/Ifelodu and Ado-Ekiti local government areas respectively. It must be clarified that these scores do not show infection or confirmed cases neither do they indicate the severity of

\section{Social Media Analysis}

The dominant themes of the Covid-19 social media messaging (Twitter) on Ekiti State starting from March to August of 2020 were more about updates and confirmation of cases. By April, the messages were about the provision of advice, and clarifications right after communication of confirmation and update. This messaging pattern suggest active Ekiti State Government leadership of the crisis communica

Ekiti Covid-19 social media discussions wer led mainly by texts made, shared, or amplified by accounts linked to government and progovernment interests.

The Covid-19 themes on social media in Ekiti State are consistent with the direction of mainstream media. Government sources (both state and federal e.g., press releases, the information distributed on social media (Twitter) and newspapers.

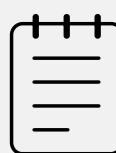

\section{News Articles Analysed}

$54 \%$ of the materials (articles, blogposts $(\mathrm{N}=790)$ were written by male authors, while $18 \%$ were written by female authors. The remaining $28 \%$ did not have any gender attributions.

The news publications $(\mathrm{N}=761)$ with the most coded extracts are The Nation ( $\mathrm{n}=$ 157: $21 \%)$, Premium Times $(n=126: 17 \%)$, The Sun $(n=100: 13 \%)$, and Nigerian organs are print media except Premium Times that is an online news publication. These publications provided more coverage on Covid-19 in Ekiti State during the first phase of the outbreak than the other publications.

Of the 554 coded extracts belonging to opinion leaders (interviewed) by gender attribution, $(n=318: 57 \%)$ are male actors or personalities, only $(n=56$ $(n=180: 33 \%)$ have no indicated gender attribution.

The News reports on Covid-19 privileged organisational leaders more than the opinions of private citizens. Most of the news actors interviewed were affiliated with political or government agencies. as traditional monarchs acs such youth leaders, and religious leaders have less sufficient representations in the mainstream media during the first months of the Covid-19 disease outbreak in Ekiti State. Most of the news discourse on Covid-19 in Ekiti State from March to August 2020 were in publications located in capital cities such as Lagos ( $=97: 13 \%)$, Abuja $(n=54: 7 \%)$, and Ado this is the exclusion of voices from rural

- The co-occurrence network maps of the mainstream media show that
their thematic narratives surround the subjects of updates, awareness, confirmation, and information dissemination.

- In the conversation graphs in the newspapers and other manstream media, antigovernment sentiments, partisan criticisms, and protests

Q-Methodology (Public

\section{Perceptions)}

- The public perspectives on Covid-19 in Ekiti State are clustered around Four (4) viewpoints and discourse dimensions. They are: (i) Distributive engagements and Socio-economic Access (Factor I), (ii) Disease Survelllance, Intervention (Factor II), (iii) Palliatives and social supports issues (Factor III) and (iv) Crisis communication, collaborative response, and protests (Factor IV). These Factors described the grouped perspectives expressed by the participants in the Q-sorting. Their pattern of aggregating the opinions in Ekiti Covid-19 conversation space.

- There are 37 opinions (Statements) in the conversation space. They are collected into matic categories.

The disease surveillance policy measure by Ekit State during the timeframe of the investigation was the Forward contact tracing approach. This entalled the isolation of known cases to forestal further transmission. However, this approach (Criteria I: Disease surveillance and face covering).

- There was a noteworthy acknowledgement of restrictions, random testing protocol and the expansion of Ekiti State's only isolation centre on the mitigation of the spread of the virus (Criteria II: Movement restriction and testing).

- The collaboration between state and nonstate local actors was not sufficient enough to significantly impact the management of Covid-19 in Ekiti State during the first phase of

Regular updates, clarifications and case confirmations were provided by the Ekiti misinformation. Awareness and information campaigns strategy of the state government aimed at bus and tricycle drivers on social and physical distancing helped stemmed the spread of the virus during the first phase of the pandemic updates, and awareness).

There are views that provision and distribution of palliatives during the also concerns about the transparency in the distribution of welfare support packages and palliatives (Criteria Palliative, social, and welfare support).

Despite government directives on social and physical distancing, many businesses and markets operate Ekiti State. Economic sustenance is primary reason for this infraction.

Education access was also was hindered by the lockdown. The alternatives provided such as the use of internet technologies, radio and television could not fully ameliorate the access limitation because of some of the factors discussed earlier including digital literacy, inadequate virtual education teaching methods and curriculum, inadequate electricity supply etc. (Criteria V: Economic, education, and access to justice).

\section{RECOMMENDATIONS (POLICY) \\ On Social Media}

Have a more distributed messaging strategy involving identifying private or non-government social media influencers who are resident within, attracted more retweets and spread. A multi-layered crisis strategic onlin communication should be more diffused and distributed to include non-state actors with relevant influence. 
- The social media crisis communication strategy for the Ekiti State should be complementary and inclusive of traditional offline communication infrastructure due to the place of Ekiti as a low internet access/ penetration state.

- $\quad$ Although mega and macro-influencers (mainly non-resident actors with large followership) are important for mass appeal; but, since all disaster events are local, it is crucial to engage more with micro and nano-influencers (residents) because they have a more focused message process with higher relevance factor. Their inclusion in the information dissemination process by tagging, mentioning, and sharing their messages (relevant) will add to the quality and reach of the communication process.

\section{On News Media}

- Ekiti State Government should engage more with local community publications in future communication planning of the state for emergency events.

- Government communication strategy during a crisis should be reformed to be more horizontal (i.e., peer-to-peer) especially among the various responsible agencies instead of the hierarchical (command-andcontrol) structure.

- Having the different agencies own their part of the communication process is more beneficial than solely centralised, top-down information. Aside from the Ekiti Commissioner for Health, the Director of the Ekiti State Emergency Management Agency Ministry, Ekiti State University Teaching Hospital should also be empowered to participate in the communication process. This will distribute the conversation further.

- Ekiti State Ministry of Information should work with the State Union of Journalists to train journalists and reporters on inclusive journalism to accommodate the underreported voices (e.g., women, youths, and rural dwellers).

\section{On Q-Methodology (Public Perceptions)}

- In future disease surveillance interventions, bidirectional contact tracing protocol should be deployed to complement available disease surveillance infrastructure including proximity-detection digital applications. Bidirectional approach "uses "reverse-tracing" to identify the parent case who infected a known case, then continues tracing to iteratively discover other cases related to the parent. ${ }^{10}$

- More Rapid SARS-CoV-2 (COVID-19) Diagnostic Antigen Testing kits be made accessible to citizens, and we also recommend the provision of more mobile testing facilities. A collaboration with local scientists could help in the development of cheaper alternatives to the more expensive kits and facilities.

- More collaborative intervention and policy initiatives between Ekiti State Government and local non-governmental actors should be emplaced. These collaborative relationships should cut across different socio-economic segments of the society and should reflect active citizen engagements.

- Mistrust of government policies around the distribution of palliatives can be averted by the enactment of measures that will encourage distributed engagements with citizens and transparency in the distribution of provisions and palliatives. Open communication and inclusive decision making on relief distributions can also prevent hoarding and corruption on the distribution chain.

- Virtual education, internet and mobile learning pedagogies should be developed by the state. Teachers should be trained on digital teaching methods to provide education access in situations where physical lessons are non-practicable.

10 Bradshaw, W. J., Alley, E. C., Huggins, J. H., Lloyd, A. L., \& Esvelt, K. M. (2021). Bidirectional contact tracing could dramatically improve $\mathrm{CO}$ VID-19 control. Nature Communications, 12(1), 1-9, 2. 